\title{
Asenkron motorların iyileştirilmiş anahtarlamalı-GKF tabanlı alan yönlendirmeli kontrolü
}

\author{
Improved switching-EKF based field oriented control of induction motors
}

\author{
Yunus Emre Altınışık ${ }^{1}$, Rıdvan Demir ${ }^{2, *}$ (D), Murat Barut ${ }^{3}$ (D) \\ ${ }^{1,3}$ Niğde Ömer Halisdemir Üniversitesi, Elektrik Elektronik Mühendisliği Bölümü, 51240, Niğde Türkiye \\ ${ }^{2}$ Kayseri Üniversitesi, Elektrik Elektronik Mühendisliği Bölümü, 38280, Kayseri Türkiye
}

\begin{abstract}
Özet
$\mathrm{Bu}$ çalışmada hız-algılayıcısız iyileştirilmiş alan yönlendirmeli kontrol (AYK) tabanlı asenkron motor (ASM) sürücüsü sunulmuştur. Hız-algılayıcısız AYK için gerekli olan rotor akıları ve rotor mekanik hızına ek olarak yük momenti, stator direnci ve rotor direnci giriş olarak ölçülen stator akım ve gerilimlerini kullanan hafıza gereksinimi ve tasarım aşaması iyileştirilmiş anahtarlamalı genişletilmiş Kalman filtresi (A-GKF) kullanılarak kestirilmiştir. Kestirilen yük momentinin ileri beslemeli kontrolü ile AYK’nın başarımı iyileştirilmiştir. Buna ek olarak parametre değişimlerinden kaynaklanan kestirim hataları stator ve rotor dirençlerinin kestirimi ile azaltılmıştır. Önerilen hız-algılayıcısız ASM sürücü zorlayıcı senaryolar ile benzetim ortamında test edilmiştir. Elde edilen sonuçlar, iyileştirilmiş AYK tabanlı ASM sürücüsünün yüksek başarıma sahip olduğunu göstermektedir.
\end{abstract}

Anahtar kelimeler: Asenkron motor, Genişletilmiş Kalman filtresi, Alan yönlendirmeli kontrol, İleri beslemeli kontrol, Durum/parametre kestirimi

\section{Giriș}

Asenkron motorlar (ASM'ler), sağlam yapıları, düşük maliyetleri, düşük bakım gereksinimleri, güvenilirlik ve zorlu ortamlarda çalışabilme özelliklerinden dolayı değişken hız ve/veya moment kontrolüne ihtiyaç duyan endüstriyel uygulamalarda ve elektrikli araçlarda yaygın olarak kullanılmakta olup; bu motorların yüksek başarımlı kontrolü için alan yönlendirmeli kontrol (AYK) ve doğrudan moment kontrol gibi yüksek başarımlı kontrol yöntemleri literatüre tanıtılmıştır. Bununla birlikte, doğrusal olmayan modeli, çalışma koşullarıyla değişen parametreleri ve bilinmeyen yük girişleri hız-algılayıcısız ASM'lerin kontrolünü karmaşık hale getirmektedir. Hız-algılayıcısız kontrolde rotor mekanik hızı ve stator/rotor akılarının kestirilmesi gerekmektedir. Ancak, yük momentindeki değişimler sıcaklık ve frekans gibi ASM'nin çalışma koşullarına bağlı olarak stator ve rotor direncinde meydana gelen değişimler ile birlikte bu kestirimlerde bozulmalara yol açmaktadır.

ASM'lerin hız-algılayıcısız kontrolü için tam dereceli gözlemleyiciler [1], sezgisel algoritmalar [2], modele uyarlamalı sistemler (MUS) [3, 4], genişletilmiş Luenberger [5] ve kayma kipli (sliding mode) [6] gözlemleyiciler ve

\section{Abstract}

In this study, the speed-sensorless improved field oriented control (FOC)-based induction motor (IM) drive has been presented. In addition to the rotor fluxes and rotor mechanical speed required for speed-sensorless FOC, the load torque, the stator resistance, and the rotor resistance are estimated by the switching extended Kalman filter ( $\mathrm{S}$ EKF) with improved memory requirement and design stage which use the measured stator currents and voltages as inputs. The performance of FOC is improved by the feedforward control of the estimated load torque. In addition, the estimation errors caused by the parameter variations are reduced with the estimations of the stator and rotor resistances. The proposed speed-sensorless IM drive is tested by challenging scenarios in simulations. The results obtained demonstrate that the improved FOC-based IM drive has a high performance.

Keywords: Induction motor, Extended Kalman filter, Field-oriented control, Feed-forward control, State/parameter estimation

genişletilmiş Kalman filtresi (GKF) [7-9] gibi farklı model tabanlı kestiriciler/gözlemleyiciler literatüre önerilmiştir. Önerilen kestirim yöntemleri arasından GKF'ler ölçme gürültülerini dikkate alarak durum ve parametre kestirim problemine olasıl bir yaklaşım sunmaktadır.

Hız-algılayıcısız ASM sürücüleri için durum ve parametre kestirimini gerçekleştiren GKF tabanlı gözlemleyiciler incelendiğinde; AYK için gerekli olan durumlara ek olarak stator ve rotor direnci kestirimi, Barut vd. (2007)'de [10] iki farklı GKF algoritmasının “anahtarlamalı" olarak çalıştırılmasıyla, Barut vd., (2008)'de [11] iki farklı GKF algoritmasının "örgülü” şekilde çalıştırılmasıyla, Barut vd. (2012)'de [8] iki farklı ASM modelinin tek GKF algoritmasına ait girişlere ardışık anahtarlanarak gerçeklemesiyle ve son olarak, Demir ve Barut (2018)'de [7] ise GKF ve MUS'un paralel çalıştırılmasıyla kestirilmiştir.

Bilinmeyen yük momenti girişlerinin, ASM'lerin hızalgılayıcısız kontrolünde hız kontrol çevrimi üzerinde bozucu bir etkiye sahip olduğu bilinmektedir. Bu bozucu etkiyi gidermek için yük momentindeki değişimler kestirilerek hız kontrol döngüsüne ileri beslenerek AYK'nın

\footnotetext{
* Sorumlu yazar / Corresponding author, e-posta / e-mail: ridvandemir@kayseri.edu.tr (R. Demir)

Geliş / Recieved: 18.03.2021 Kabul / Accepted: 19.04.2021 Yayımlanma/Published: 27.07.2021

doi: $10.28948 /$ ngmuh. 899447
} 
başarımının iyileştirilebilir olduğu bilinmektedir. Yük momentinin kestirimi için literatürde iki farklı yaklaşım bulunmaktadır. İlk yaklaşımda, yük momentinin kestirimi kontrol sistemi için gerekli olan durumları kestiren gözlemleyiciye ek olarak harici bir kestirici/gözlemleyici tarafından gerçekleştirilmektedir [12, 13]. İkinci yaklaşımda ise yük momentinin kestirimi tek bir gözlemleyici kullanılarak kontrol sistemi için gerekli olan durumlar ile birlikte gerçekleştirilmektedir [14-16].

$\mathrm{Bu}$ çalışmanın temel katkısı stator ve rotor dirençlerinde meydana gelen değişimler ile bilinmeyen yük girişlerinin sebep olduğu bozulmayı önleyebilen yüksek kontrol başarımına sahip hız-algılayıcısız hafıza gereksinimi ve tasarım aşaması iyileştirilmiş AYK tabanlı ASM sürücüsünü literatüre tanıtmaktır. Bu amaç doğrultusunda ilk olarak hızalgılayıcısız AYK için gerekli olan rotor akısı, rotor mekanik hızı ile birlikte stator direnci, rotor direnci ve yük momentinin eş-zamanlı kestirimleri için A-GKF gözlemleyicisi önerilmiştir. Daha sonra, kestirilen yük momentinin ileri beslemeli kontrolü ile AYK'nın başarımı iyileştirilmiştir. Buna ek olarak parametre değişimlerinden kaynaklanan kestirim hataları stator ve rotor dirençlerinin kestirimi ile azaltılmıştır. Böylece tek bir gözlemleyici kullanarak AYK tabanlı ASM sürücüsünün başarımı önemli ölçüde artırılmıştır. Önerilen hız-algılayıcısız ASM sürücüsü zorlayıcı senaryolar ile benzetim ortamında test edilmiştir. Elde edilen sonuçlar, iyileştirilmiş AYK tabanlı ASM sürücüsünün ve A-GKF gözlemleyicisinin yüksek başarıma sahip olduğunu göstermektedir.

\section{ASM'nin matematiksel modeli}

ASM'lerin matematiksel modeli aşağıdaki verilen genel formda ifade edilebilir.

$$
\begin{gathered}
\dot{\mathbf{x}}_{k}=\mathbf{f}\left(\mathbf{x}_{k}, \mathbf{u}_{k}\right)+\mathbf{w}_{k} \\
\mathbf{z}_{k}=\mathbf{h}\left(\mathbf{x}_{k}\right)+\mathbf{v}_{k}
\end{gathered}
$$

Burada, f, durum ve girişlerin doğrusal olmayan fonksiyonu; $\mathbf{x}_{k}$ durum vektörü; $\mathbf{u}_{k}$ kontrol giriş vektörü; $\mathbf{h}$ çıkışların fonksiyonu; $\mathbf{w}_{k}$ ve $\mathbf{v}_{k}$ ise sırası ile süreç ve ölçüm gürültüleri olarak tanımlanabilir.

Genel formu Denklem (1)'de verilen ASM modelindeki vektörler aşağıda verilmiştir.

$$
\begin{aligned}
& \mathbf{x}_{k}=\left[\begin{array}{lllll}
i_{s \alpha} & i_{s \beta} & \psi_{r \alpha} & \psi_{r \beta} & \omega_{m}
\end{array}\right]^{T} \\
& \mathbf{u}_{t}=\left[\begin{array}{ll}
v_{s \alpha} & v_{s \beta}
\end{array}\right]^{T}, \quad \mathbf{h}=\left[\begin{array}{ll}
i_{s \alpha} & i_{s \beta}
\end{array}\right]^{T} \\
& \mathbf{f}=\left[\begin{array}{c}
-\left(\frac{R_{s}}{L_{\sigma}}-\frac{R_{r} L_{m}^{2}}{L_{\sigma} L_{r}^{2}}\right) i_{s \alpha}+\frac{R_{r} L_{m}}{L_{\sigma} L_{r}^{2}} \psi_{r \alpha}+\frac{L_{m} p_{p}}{L_{\sigma} L_{r}} \omega_{m} \psi_{r \beta}+\frac{v_{s \alpha}}{L_{\sigma}} \\
-\left(\frac{R_{s}}{L_{\sigma}}-\frac{R_{r} L_{m}^{2}}{L_{\sigma} L_{r}^{2}}\right) i_{s \beta}+\frac{R_{r} L_{m}}{L_{\sigma} L_{r}^{2}} \psi_{r \beta}-\frac{L_{m} p_{p}}{L_{\sigma} L_{r}} \omega_{m} \psi_{r \alpha}+\frac{v_{s \beta}}{L_{\sigma}} \\
\frac{R_{r} L_{m}}{L_{r}} i_{s \alpha}-\frac{R_{r}}{L_{r}} \psi_{r \alpha}-p_{p} \omega_{m} \psi_{r \beta} \\
\frac{R_{r} L_{m}}{L_{r}} i_{s \beta}-\frac{R_{r}}{L_{r}} \psi_{r \beta}+p_{p} \omega_{m} \psi_{r \alpha} \\
\frac{3 p_{p} L_{m}}{2 J_{t} L_{r}}\left(\psi_{r \alpha} i_{s \beta}-\psi_{r \beta} i_{s \alpha}\right)-\frac{B_{t}}{J_{t}} \omega_{m}-\frac{\tau_{l}}{J_{t}}
\end{array}\right]
\end{aligned}
$$

Burada $v_{s \alpha}, \quad v_{s \beta}, i_{s \alpha}$ ve $i_{s \beta}$ stator duran eksen takımındaki stator gerilim ve akımlarının bileşenlerini; $\psi_{r \alpha}$ ve $\psi_{r \beta}$ stator duran eksen takımındaki rotor akılarının bileşenlerini; $R_{r}$ ve $R_{S}$ rotor ve stator dirençlerini; $L_{r}, L_{s}$ ve $L_{m}$ rotor, stator ve mıknatıslama endüktanslarını; $L_{\sigma}=$ $\sigma L_{s}=L_{s}-L_{m}^{2} / L_{r}$, stator geçici endüktansını; $\sigma$ kaçak faktörünü; $\omega_{m}$ rotor mekanik açısal hızını; $\tau_{l}$ yük momentini; $p_{p}$ kutup çifti sayısını; $J_{t}$ ve $B_{t}$ sırasıyla ASM ve yükün toplam eylemsizliği ile toplam viskoz sürtünme terimini; $p_{p}$ ise kutup çifti sayısını ifade etmektedir.

\section{Anahtarlamalı GKF'nin tasarımı}

GKF ile $i_{s \alpha}, i_{s \beta}, \psi_{r \alpha}, \psi_{r \beta}, \omega_{m}, \tau_{l}, R_{r}$ ve $R_{s}$ 'nin eşzamanlı kestirimini gerçekleştirmek için gözlemleyicide kullanılan ASM modeli; genel formu (1)'de verilen ASM modelinin $\tau_{l}$ ve $R_{r} / R_{S}$ ile genişletilmesi ile oluşturulmuştur. Genişletilmiş modelde $\tau_{l}$ ve $R_{r} / R_{s}$ kestirilecek olan parametre olarak göz önünde bulundurulmaktadır. Gözlemleyicide kullanılan genişletilmiş ASM modeline ait vektörler aşağıda verilmiştir.

$$
\begin{aligned}
& \mathbf{x}_{o, k}=\left[\begin{array}{lllllll}
i_{s \alpha} & i_{s \beta} & \psi_{r \alpha} & \psi_{r \beta} & \omega_{m} & \tau_{l} & R_{r} / R_{s}
\end{array}\right]^{T}, \\
& \mathbf{u}_{o, k}=\left[\begin{array}{ll}
v_{s \alpha} & v_{s \beta}
\end{array}\right]^{T}, \mathbf{h}_{o}=\left[\begin{array}{ll}
i_{s \alpha} & i_{s \beta}
\end{array}\right]^{T}, \\
& \mathbf{f}_{o}=\left[\begin{array}{c}
-\left(\frac{R_{s}}{L_{\sigma}}-\frac{R_{r} L_{m}^{2}}{L_{\sigma} L_{r}^{2}}\right) i_{s \alpha}+\frac{R_{r} L_{m}}{L_{\sigma} L_{r}^{2}} \psi_{r \alpha}+\frac{L_{m} p_{p}}{L_{\sigma} L_{r}} \omega_{m} \psi_{r \beta}+\frac{v_{s \alpha}}{L_{\sigma}} \\
-\left(\frac{R_{s}}{L_{\sigma}}-\frac{R_{r} L_{m}^{2}}{L_{\sigma} L_{r}^{2}}\right) i_{s \beta}+\frac{R_{r} L_{m}}{L_{\sigma} L_{r}^{2}} \psi_{r \beta}-\frac{L_{m} p_{p}}{L_{\sigma} L_{r}} \omega_{m} \psi_{r \alpha}+\frac{v_{s \beta}}{L_{\sigma}} \\
\frac{R_{r} L_{m}}{L_{r}} i_{s \alpha}-\frac{R_{r}}{L_{r}} \psi_{r \alpha}-p_{p} \omega_{m} \psi_{r \beta} \\
\frac{R_{r} L_{m}}{L_{r}} i_{s \beta}-\frac{R_{r}}{L_{r}} \psi_{r \beta}+p_{p} \omega_{m} \psi_{r \alpha} \\
\frac{3 p_{p} L_{m}}{2 J_{t} L_{r}}\left(\psi_{r \alpha} i_{s \beta}-\psi_{r \beta} i_{s \alpha}\right)-\frac{\tau_{l}}{J_{t}} \\
0 \\
0
\end{array}\right]
\end{aligned}
$$

Denklem (2)'de verilen ileri Euler yaklaşımının kullanılması ile gözlemleyicide kullanılan ayrık zamanlı ASM modeli Denklem (3)’teki gibi verilir.

$$
\begin{gathered}
\dot{\boldsymbol{x}}_{k} \approx \frac{\boldsymbol{x}_{k+1}-\boldsymbol{x}_{k}}{T} \\
\mathbf{x}_{o, k+1}=\mathbf{I}_{7 \times 7} \times \mathbf{x}_{o, k}+T \times \mathbf{f}_{o}
\end{gathered}
$$

Burada I birim matris ve $T$ örnekleme zamanıdır.

$i_{s \alpha}, i_{s \beta}, \psi_{r \alpha}, \psi_{r \beta}, \omega_{m}, \tau_{l}, R_{r}$ ve $R_{s}$ 'nin eş-zamanlı kestirimini gerçekleştiren GKF algoritmasına ilişkin geleneksel denklemler aşağıda verilmiştir.

- Doğrusallaştırma

$$
\mathbf{F}_{\mathrm{k}+1 \mid \mathrm{k}}=\left.\frac{\partial \mathbf{f}(\mathbf{x}, \mathbf{u})}{\partial \mathbf{x}}\right|_{\mathbf{x}=\hat{\mathbf{x}}_{\mathrm{k}+1}}
$$

- Kestirim veya zaman güncellemesi

$$
\hat{\mathbf{x}}_{\mathrm{k}+1}^{-}=\mathbf{f}\left(\hat{\mathbf{x}}_{\mathrm{k}}, \mathbf{u}_{\mathrm{k}+1}\right)
$$




$$
\mathbf{P}_{\mathrm{k}+1}^{-}=\mathbf{F}_{\mathrm{k}+1} \mathbf{P}_{\mathrm{k}} \mathbf{F}_{\mathrm{k}+1}^{\mathrm{T}}+\mathbf{Q}
$$

- Ölçüm veya düzeltme güncellemesi

$$
\begin{gathered}
\mathbf{K}_{\mathrm{k}+1}=\mathbf{P}_{\mathrm{k}+1}^{-} \mathbf{H}_{\mathrm{k}+1}^{\mathrm{T}}\left[\mathbf{H}_{\mathrm{k}+1} \mathbf{P}_{\mathrm{k}+1}^{-} \mathbf{H}_{\mathrm{k}+1}^{\mathrm{T}}+\mathbf{R}\right]^{-1} \\
\hat{\mathbf{x}}_{\mathrm{k}+1}=\hat{\mathbf{x}}_{\mathrm{k}+1}^{-}+\mathbf{K}_{\mathrm{k}+1}\left(\mathrm{z}_{\mathrm{k}+1}-\mathbf{H}_{\mathrm{k}+1} \hat{\mathbf{x}}_{\mathrm{k}+1}^{-}\right) \\
\mathbf{P}_{\mathrm{k}+1}=\mathbf{P}_{\mathrm{k}+1}^{-}-\mathbf{K}_{\mathrm{k}+1} \mathbf{H}_{\mathrm{k}+1} \mathbf{P}_{\mathrm{k}+1}^{-}
\end{gathered}
$$

Burada $\mathbf{F}_{k+1 \mid k}$ doğrusal olmayan modeli doğrusallaştırmada kullanılan fonksiyonu; $\mathbf{P}_{k+1}^{-}$ve $\mathbf{P}_{\mathrm{k}+1}$ sirasıyla hata kovaryans matrisinin önceki ve sonraki değerlerini; $\mathbf{K}_{k+1}$ Kalman kazancını; $\mathbf{Q}$ sistem gürültüsünün kovaryans matrisini; $\mathbf{R}$ ise çıkış gürültüsünün kovaryans matrisini ifade etmektedir. Bu çalışmada durum ve parametre kestiriminde kullanılan genel şeması Şekil 1'de verilen hafıza gereksinimi ve tasarım aşaması iyileştirilmiş A-GKF algoritması Denklem (4)-(9) incelenerek aşağıdaki yorumlar altında oluşturulmuştur.

Yorum 1: Denklem (5)-(9) GKF'nin kestirim ve düzeltme adımlarını oluşturur.

Yorum 2: Denklem (5)-(9)'da f, F ve $\mathbf{Q}$ matrislerinde sadece $R_{r}$ ve $R_{s}$ 'ye bağlı olarak üretilen girişler anahtarlanmaktadır.

Önerilen A-GKF algoritmas1, $i_{s \alpha}, i_{s \beta}, \psi_{r \alpha}, \psi_{r \beta}, \omega_{m}, \tau_{l}$, $R_{r}$ ve $R_{S}$ 'nin eş-zamanlı kestirimini; iki GKF algoritmasının anahtarlamalı çalıştırılması ile gerçekleştiren Barut vd. (2007) [10] ve Barut vd. (2008) [11] ve iki ASM modelinin tek bir GKF gövdesinin girişinde anahtarlanması ile gerçekleştiren Barut vd. (2012) [8]'den farklı olarak tek bir ASM modelinin içerisinde sadece $R_{r}$ ve $R_{s}$ 'ye bağlı olarak üretilen girişlerin anahtarlanması ile gerçekleştirmektedir. Her bir anahtarlama periyodunda kestirilen $i_{s \alpha}, i_{s \beta}, \psi_{r \alpha}$, $\psi_{r \beta}, \omega_{m}$, ve $\tau_{l}$ 'ye ilave olarak $R_{r}$ ve $R_{s}$ 'den yalnız bir tanesi kestirilir. $R_{r}$ ya da $R_{S}$ 'nin kestirilmiş değeri diğer direnç kestiriminin gerçekleştirildiği periyodda GKF algoritmasının girişinde sabit parametre olarak kullanılır. Böylece önerilen A-GKF algoritması, $i_{s \alpha}, i_{s \beta}, \psi_{r \alpha}, \psi_{r \beta}, \omega_{m}$, $\tau_{l}, R_{r}$ ve $R_{s}$ 'nin eş-zamanlı kestirimini;

- iki GKF algoritmasının anahtarlamalı çalıştırılması ile gerçekleştiren Barut vd. (2007) [10] ve Barut vd. (2008) [11] ile,

- iki ASM modelinin tek bir GKF gövdesinin girişinde anahtarlandığı Barut vd. (2012) [8] ile

kıyaslandığında hafiza gereksinimi ve zaman alıcı tasarım aşamaları iyileştirilmiş olmaktadır.

\section{4 İyileştirilmiş hız-algılayıcısız AYK'lı ASM sürücüsü}

Geleneksel AYK'dan farklı olarak hız kontrolünü iyileştirmek için kestirilen yük momenti hı kontrol çevrimine dahil edildiği iyileştirilmiş AYK blok şeması Şekil 2'de gösterilmiştir. Şekil 2'de verilen bütün kontrolörler PI tipi kontrolördür ve $\hat{\theta}_{r f}$ stator duran eksenine göre rotor akısının açısal konumudur. Hız-algılayıcısız AYK için gerekli olan rotor hızı ve rotor akısı ile birlikte yük momenti, rotor direnci ve stator direncinin kestirimi bu çalışmada önerilen A-GKF gözlemleyicisi tarafından gerçekleştirilmektedir.

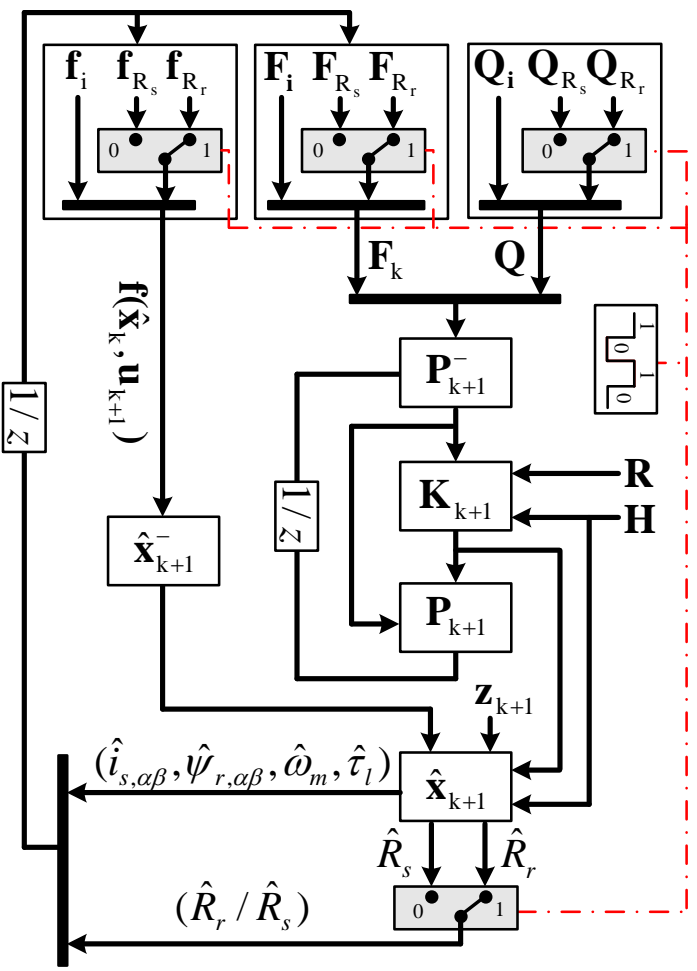

Şekil 1. İyileştirilmiş A-GKF algoritmasının blok şeması

\section{Benzetim çalışmaları}

Önerilen AYK tabanlı ASM sürücüsü MATLAB/Simulink ortamında tasarlanmıştır. Benzetim çalışmalarında kullanılan ASM'nin anma değerleri ve parametreleri Tablo 1'de verilmiştir. Benzetim çalışmalarında örnekleme zamanı (T) $100 \mu \mathrm{s}$ olarak seçilmiştir. ASM'lerde rotor direncinin değeri stator direncine göre daha doğru belirlenemediği için GKF gözlemleyici ilk olarak stator direncinin anma değeri ile başlatılıp $R_{r}$ kestirimi gerçekleştirilmiştir. Daha sonra, $t=0.2 s^{\prime}$ de $R_{r}$ ve $R_{s}$ 'ye bağlı olarak üretilen girişler her $1 \times T$ 'de bir anahtarlanarak kestirimler eş-zamanlı olarak gerçekleştirilmiştir.

Tablo 1. ASM'nin anma değerleri ve parametreleri [17]

\begin{tabular}{cc||cc}
\hline$P[\mathrm{~kW}]$ & 3 & $p_{p}$ & 2 \\
$f[\mathrm{~Hz}]$ & 50 & $R_{s}[\Omega]$ & 2.283 \\
$V[\mathrm{~V}]$ & 380 & $R_{r}[\Omega]$ & 2.133 \\
$I[\mathrm{~A}]$ & 6.9 & $L_{s}[\mathrm{H}]$ & 0.2311 \\
$n_{m}[\mathrm{dev} / \mathrm{dk}]$ & 1430 & $L_{r}[\mathrm{H}]$ & 0.2311 \\
$\tau_{l}[\mathrm{~N} . \mathrm{m}]$ & 20 & $L_{m}[\mathrm{H}]$ & 0.22 \\
\hline
\end{tabular}

A-GKF algoritmasının başarımını etkileyen ve deneme yanılma yöntemi ile belirlenen gürültü kovaryans matrisleri ( $\mathbf{Q}$ ve $\mathbf{R}$ ) ile hata kovaryans matrisinin $\left(\mathbf{P}_{0}\right.$ '’n) başlangıç değerleri aşağıdaki gibidir:

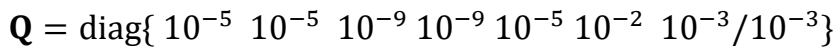

$\mathbf{R}=\operatorname{diag}\left\{\begin{array}{ll}10^{-6} & 10^{-6}\end{array}\right\}$

$\mathbf{P}_{0}=\operatorname{diag}\left\{\begin{array}{lllllll}10 & 10 & 10 & 10 & 10 & 10 & 10 / 10\end{array}\right.$ 


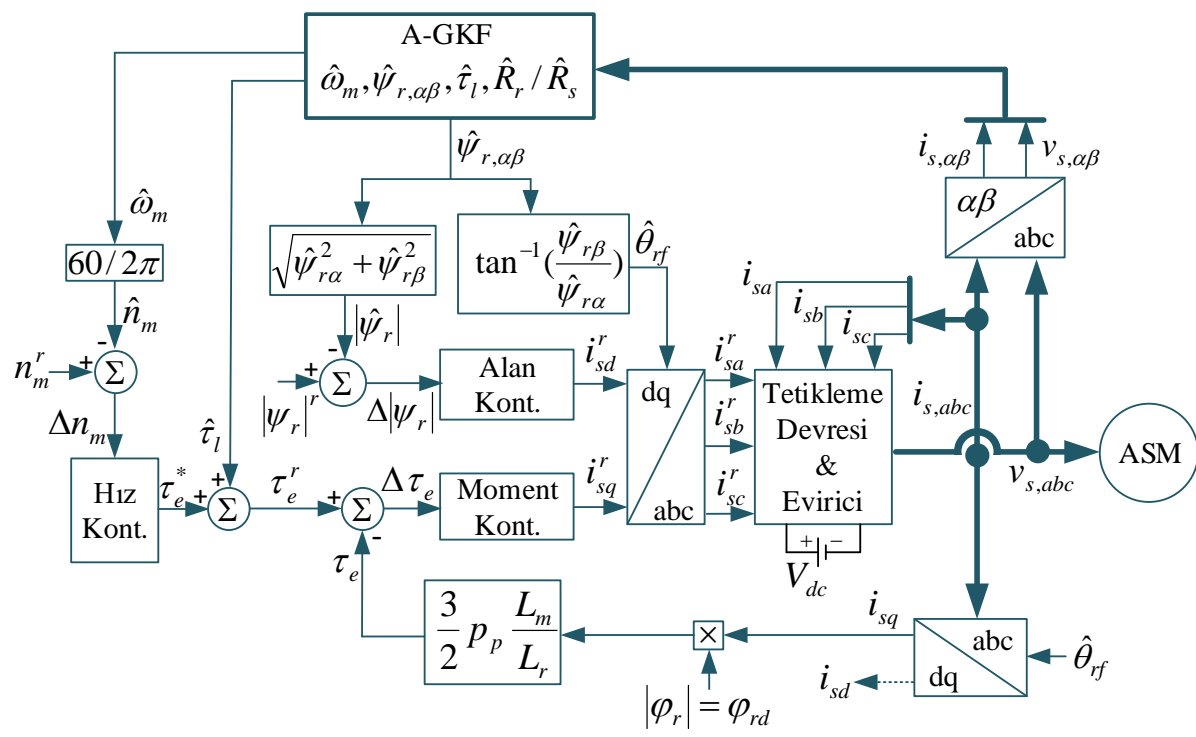

Şekil 2. Önerilen hız-algılayıcısız AYK tabanlı ASM sürücüsünün blok şeması

Önerilen AYK tabanlı ASM sürücüsünün başarımını göstermek için, yük momenti değişimleri altında yük momentinin ileri beslemeli kontrolünün (IBK'nın) dahil edildiği önerilen AYK tabanlı sürücü sistemi ve dahil edilmediği geleneksel AYK tabanlı sürücü sistemi için karşılaştırmalı sonuçlar verilmiştir. Her iki sürücü sisteminin başarımını test etmek için üretilen senaryolar aşağıda verilmiştir:

- İlk senaryoda sıfir hız bölgesinde yük momenti, rotor direnci ve stator direnci değişimleri altında ASM sürücülerinin kontrol başarımları ve A-GKF gözlemleyicisinin kestirim başarımı test edilmiştir.

- İkinci senaryoda anma hız bölgesinde yük momenti, rotor direnci ve stator direnci değişimleri altında ASM sürücülerinin kontrol başarımları ve A-GKF gözlemleyicisinin kestirim başarımı test edilmiştir.

- Son olarak IBK'lı AYK tabanlı ASM sürücüsünün başarımı geniş bir hız aralığında test edilmiştir.

AYK tabanlı ASM sürücülerinden elde edilen karşılaştırmalı benzetim sonuçları Şekil 3-6'da, geniş bir hız aralığında test edilen IBK'lı AYK tabanlı ASM sürücüsünden elde edilen sonuçlar ise Şekil 7'de verilmiştir.

Şekil 3, 5 ve 7'de kullanılan " $m$ " ve " $r e f$ " üst indisleri sırasıyla ölçülen büyüklük ve referans değişimleri, “ $\widehat{*}$ " A-GKF gözlemleyici tarafindan kestirilen durum ve parametreleri, Şekil 4, 6 ve 7'de ise “ $e_{(*)}$ ” kestirilen durum ve parametreler ile referans değişimler arasındaki hatayı ifade etmektedir.

\subsection{Senaryo I}

$\mathrm{Bu}$ senaryoda önerilen hız-algılayıcısız AYK tabanlı ASM sürücüsünün başarımı, sıfır hız bölgesinde yük momenti, rotor direnci ve stator direnci değişimleri altında test edilmiştir. Önerilen sürücü sisteminin başarımı kestirilen yük momentinin kontrol sisteminde hız çevrimine dahil edilmediği sürücü sistemi ile karşılaştırmalı olarak sunulmuştur. $\mathrm{Bu}$ amaç için, ASM sıfır ile yüksüz olarak sürülürken sırasıyla ve basamak biçiminde $t=1 \mathrm{~s}$ 'de yük momenti anma değerine, $t=2 s$ 'de rotor direnci anma değerinin iki katına son olarak $\mathrm{t}=3 \mathrm{~s}$ 'de stator direnci anma değerinin iki katına çıkarılmıştır. Her iki sürücü sistemine ait sonuçlar Şekil 3 ve 4'te verilmiştir. Her iki sürücü sistemi de yük momenti, rotor ve stator direncinde meydana getirilen değişimlerin üstesinden gelse de, yük momentinin hız çevrimine ileri beslendiği sürücü sisteminde yük momentinin basamak değişimi ile meydana gelen geçici durum, geleneksel AYK tabanlı ASM sürücüsüne göre $\% 90$ daha kisadir.

\subsection{Senaryo II}

$\mathrm{Bu}$ senaryoda önerilen hız-algılayıcısız AYK tabanlı ASM sürücüsünün başarımı, anma hız bölgesinde basamak şeklinde meydana getirilen yük momenti, rotor direnci ve stator direnci değişimleri altında test edilmiştir. Her iki sürücü sisteminin kontrol başarımları Şekil 5 ve 6'da verilmiştir. İlk senaryoya benzer şekilde yük momentinin hız çevrimine ileri beslendiği hız-algılayıcısız AYK tabanlı ASM sürücüsünde yük momentinin değişimi ile meydana gelen $\omega_{m}$ 'deki geçici durum geleneksel AYK tabanlı ASM sürücüsüne göre daha kısadır. Ayrıca, anma hızında rotor ve stator direnci değişimlerine karşı her iki sürücü de yüksek başarıma sahiptir.

\subsection{Senaryo III}

$\mathrm{Bu}$ senaryoda önerilen iyileştirilmiş hız-algılayıcısız IBK'lı AYK tabanlı ASM sürücüsünün başarımı, sıfır hız, anma hızı ve hı terslendirmesini içeren geniş bir hız bölgesinde basamak ve/veya doğrusal şeklinde üretilen yük momenti, rotor direnci ve stator direnci değişimleri altında test edilmiştir. A-GKF ile iyileştirilmiş hı-algılayıcısız AYK tabanlı ASM sürücü sisteminin kontrol başarımı Şekil 7'de verilmiştir. Elde edilen sonuçlar önerilen AYK tabanlı sürücü sisteminin yük ve direnç değişimleri altında her iki yönde de yüksek bir başarıma sahip olduğunu onaylamaktadir. 

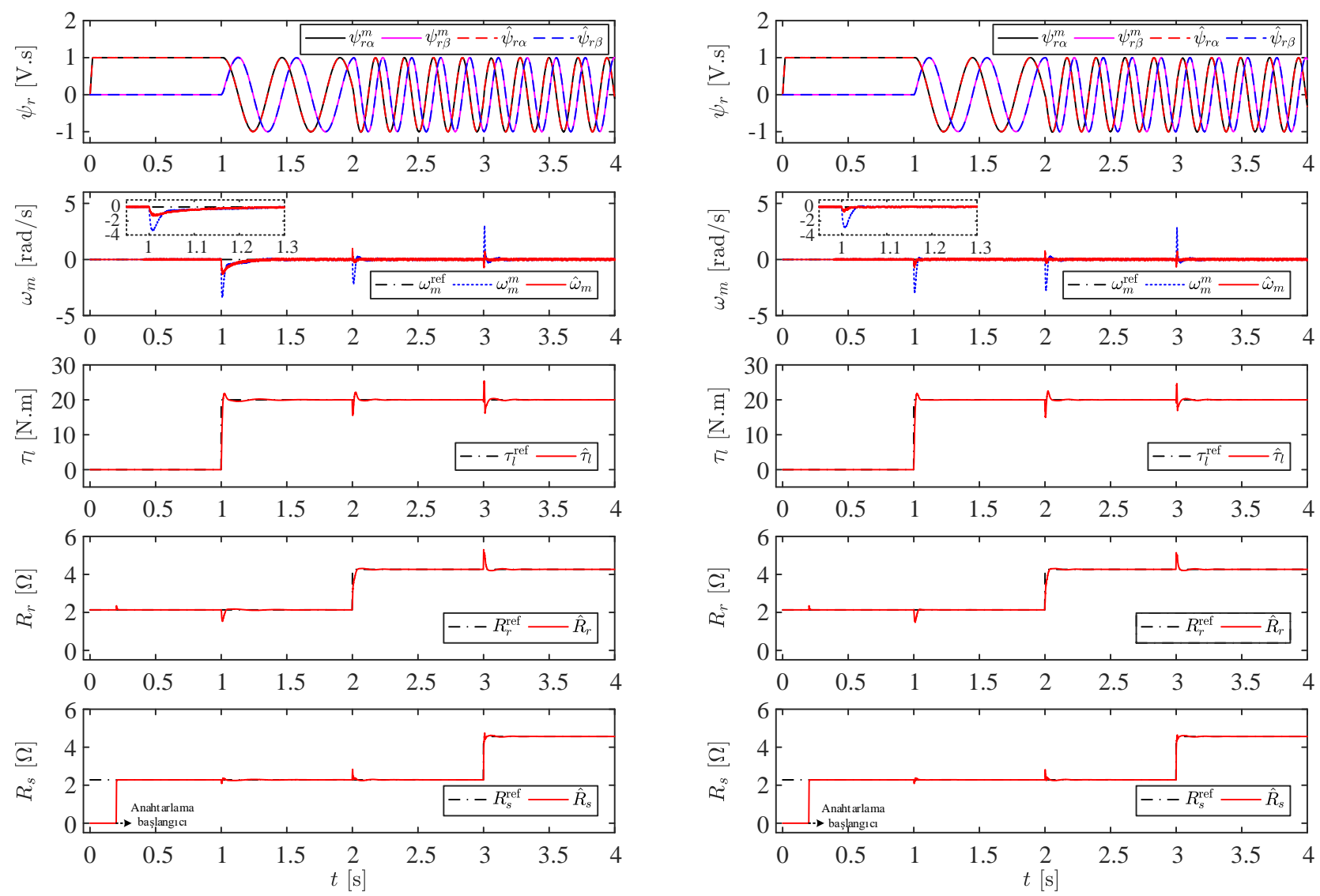

(a)

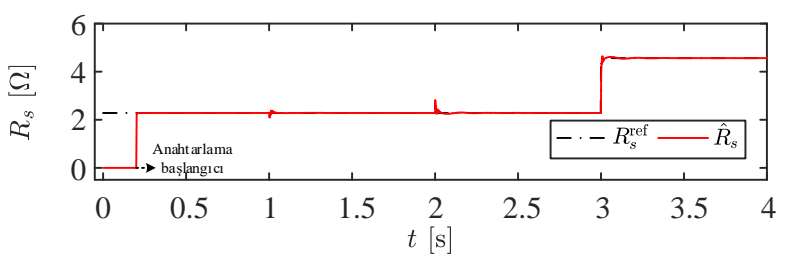

(b)

Şekil 3. Sıfır hızda ASM sürücüsünün kontrol ve A-GKF'nin kestirim başarımları (a) IBK'sı AYK ve (b) IBK'lı AYK
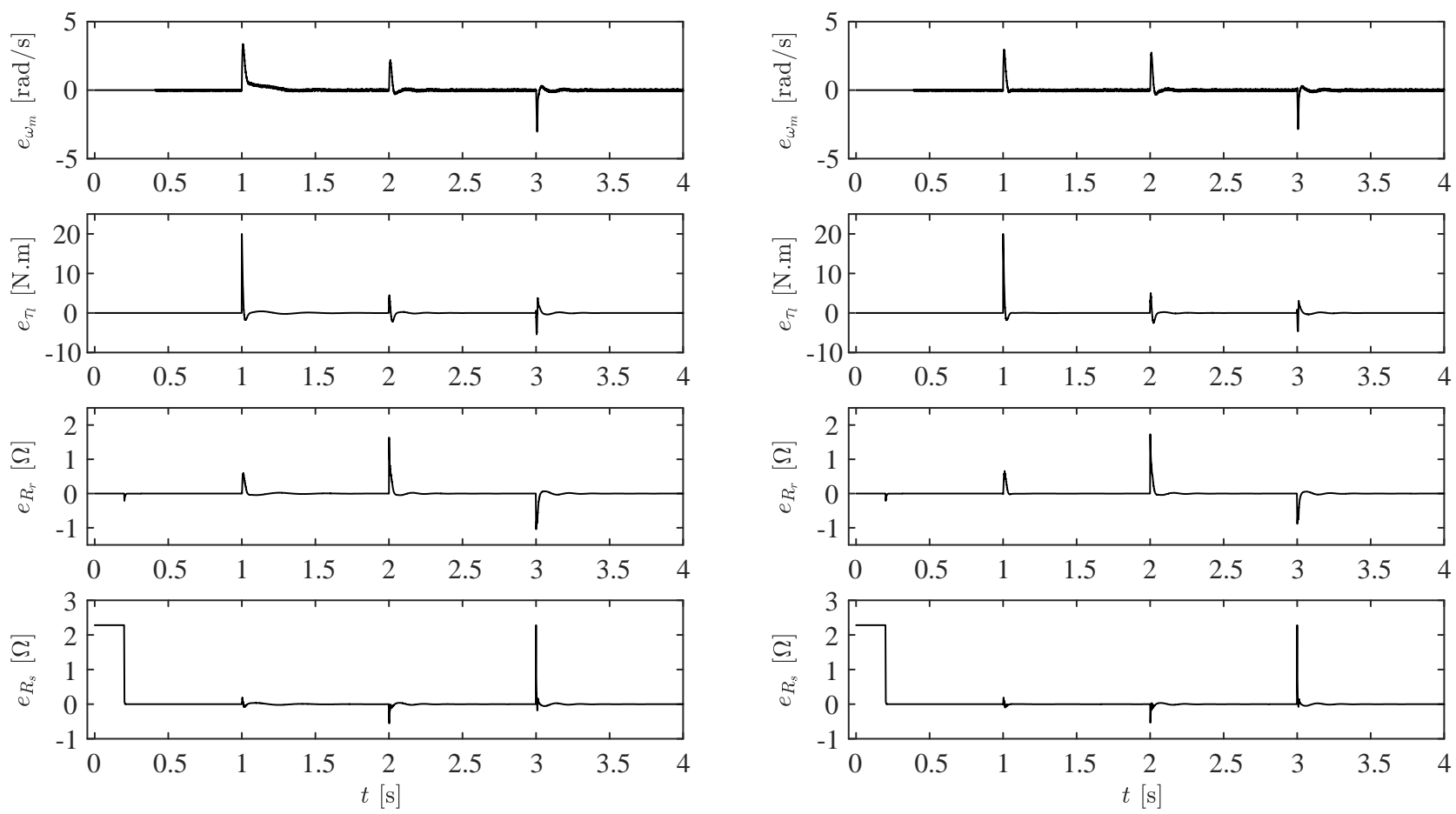

(a)

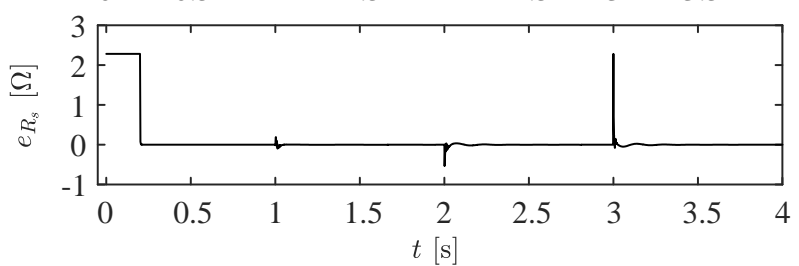

(b)

Şekil 4. Sıfır hızda ASM sürücüsünün ve A-GKF'nin izleme ve kestirim hataları (a) IBK'sız AYK ve (b) IBK'lı AYK 

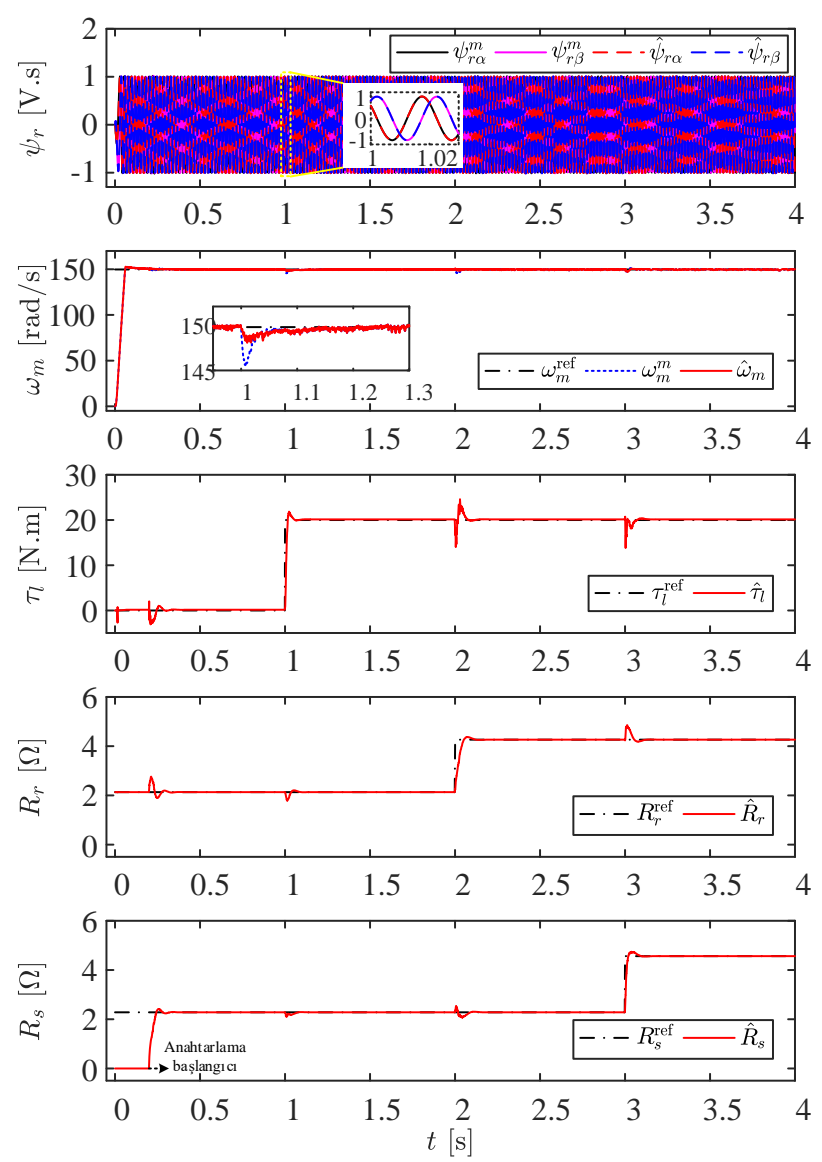

(a)
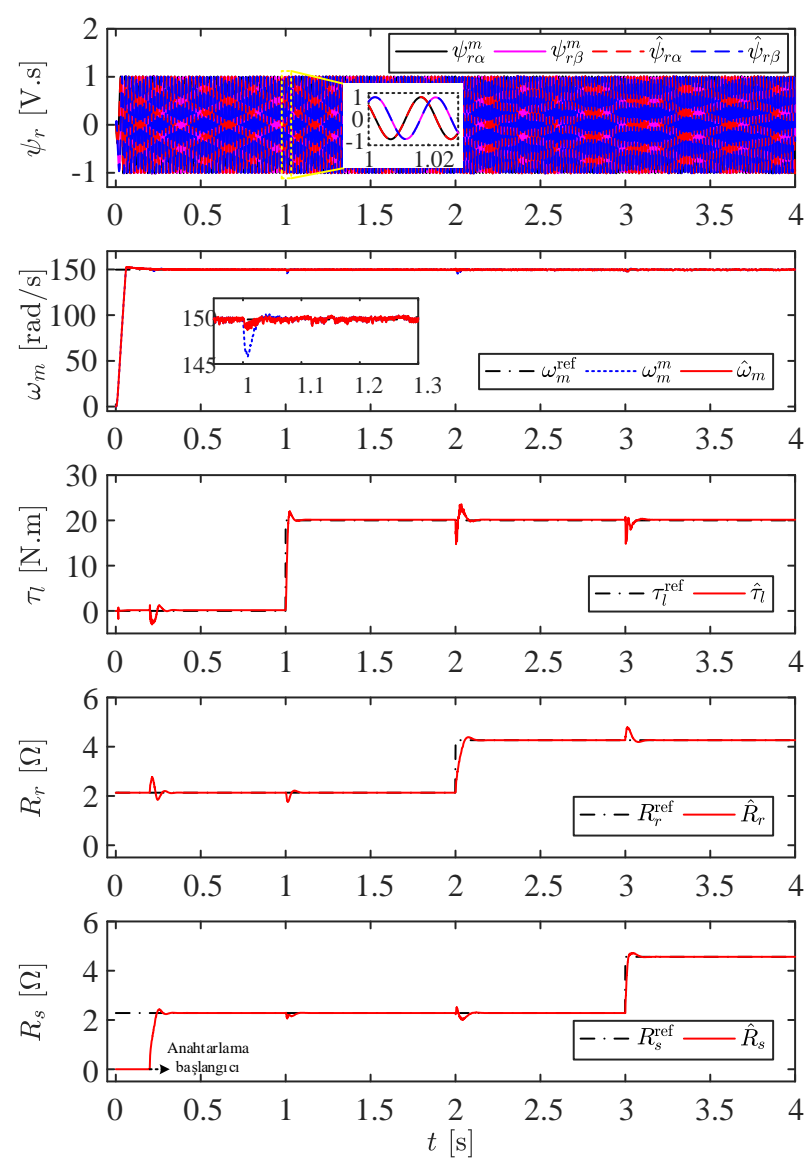

(b)

Şekil 5. Anma hızda ASM sürücüsünün kontrol ve A-GKF’nin kestirim başarımları (a) IBK'sız AYK ve (b) IBK'lı AYK
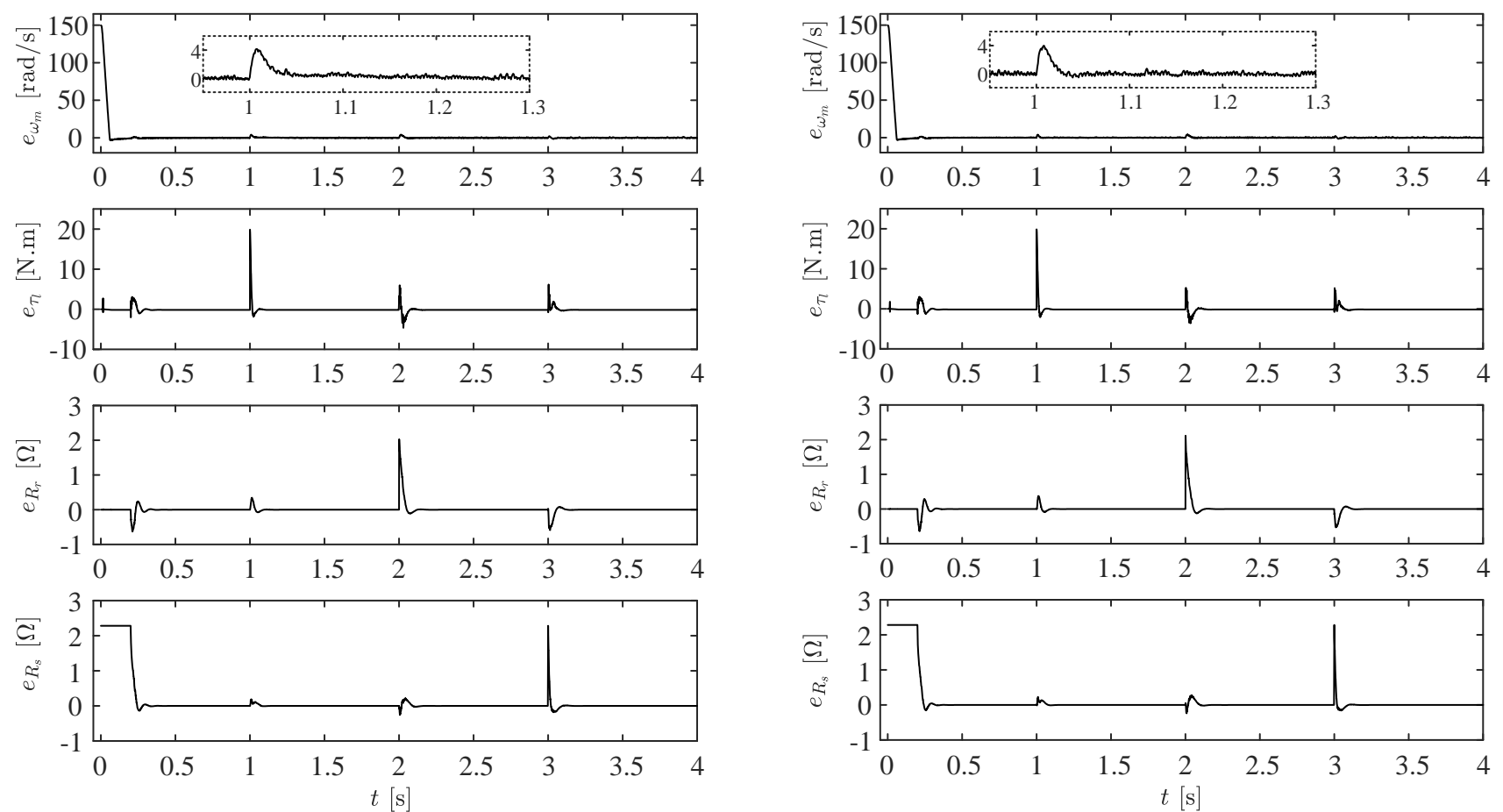

(a)

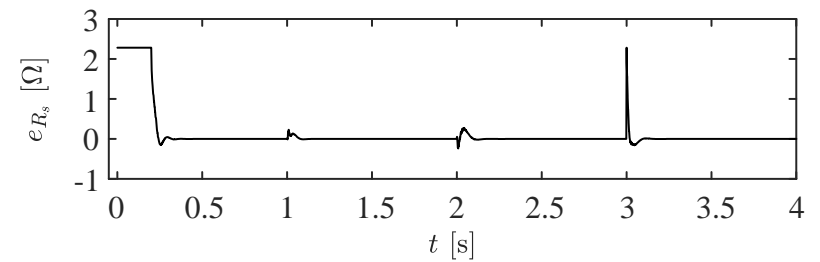

(b)

Şekil 6. Anma hızda ASM sürücüsünün ve A-GKF'nin izleme ve kestirim hataları (a) IBK'sı AYK ve (b) IBK'lı AYK 

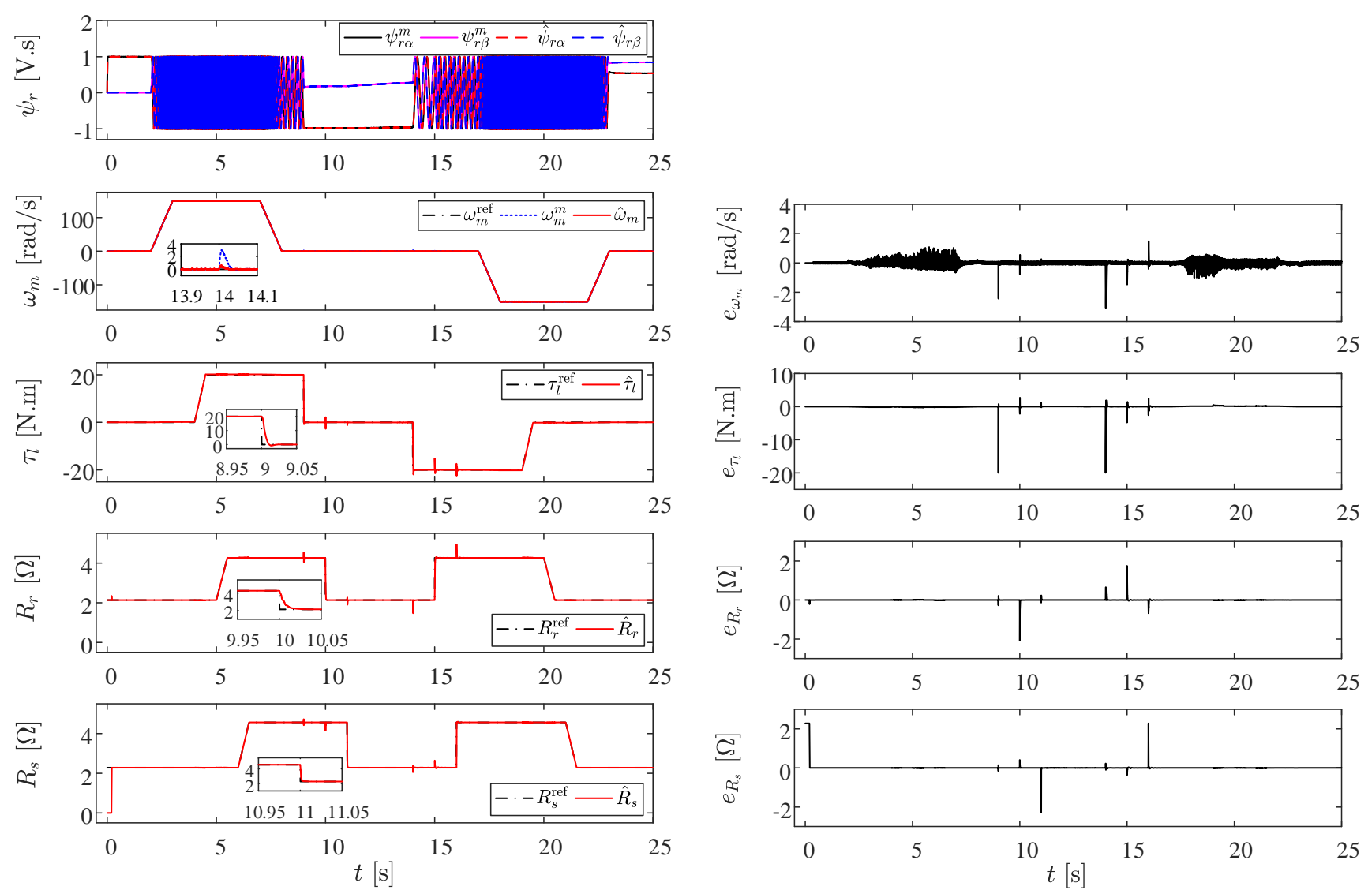

(a)

(b)

Şekil 7. IBK'lı AYK tabanlı ASM sürücüsünün kontrol başarımı (a) Kestirim ve kontrol başarımı (b) Kestirim ve izleme hataları

Benzetim çalışmalarından elde edilen sonuçlar (Şekil 37) hem sıfır hız hem de anma hızı bölgelerinde, bu çalışmada önerilen A-GKF kestirim algoritmasının ve yük momentinin hız çevrimine İBK'sı ile iyileştirilen AYK sisteminin yüksek başarıma sahip olduğu onaylamaktadır. Ayrıca, Şekil 3 ASM'lerin durum ve parametre kestirimi için doğru akım (DA) koşulu [18] $(0 s \leq t \leq 1 s)$ olarak bilinen en kötü senaryoyu içermektedir. Bu zorlayıcı DA koşuluna rağmen önerilen A-GKF algoritmasının kestirim ve AYK tabanlı ASM sürücüsünün kontrol başarımları oldukça iyidir.

Özetle yalnızca ölçülen stator akım ve gerilimlerini kullanan ve bu çalışmada önerilen A-GKF ile, AYK için gerekli olan $\psi_{r \alpha}, \psi_{r \beta}, \omega_{m}$ durumları ile birlikte $\tau_{l}, R_{r}$ ve $R_{s}$ parametreleri eş-zamanlı olarak yüksek bir doğrulukla sıfır hız, anma hızı ve hız terslendirmelerini içeren geniş bir hız aralığında benzetim temelli olarak kestirilebilmektedir.

\section{Sonuçlar}

$\mathrm{Bu}$ çalışmada hız-algılayıcısız hafıza gereksinimi ve tasarımı iyileştirilmiş A-GKF'yi kullanan AYK tabanlı bir ASM sürücüsü önerilmiştir. Ölçülen stator akım ve gerilimlerini kullanarak hız-algılayıcısız AYK için gerekli olan rotor akıları ve rotor mekanik hızına ek olarak yük momenti, stator direnci ve rotor direnci önerilen A-GKF ile kestirilmiştir. Böylece bilinmeyen yük momenti, stator ve rotor dirençlerinin değişimlerinin kestirim ve kontrol sisteminde meydana getirdiği bozulmalar giderilmiştir. Önerilen hız-algılayıcısız ASM sürücüsü zorlayıc1 senaryolar ile benzetim ortamında test edilmiştir. Elde edilen sonuçlar, iyileştirilmiş AYK tabanlı ASM sürücüsünün yüksek başarıma sahip olduğunu göstermektedir.

\section{Çıkar çatışması}

Yazar çıkar çatışması olmadığını beyan etmektedir.

Benzerlik oranı (iThenticate): $\% 8$

\section{Kaynaklar}

[1] S. Yin, Y. Huang, Y. Xue, D. Meng, C. Wang, Y. Lv, L. Diao and J. Jatskevich, Improved full-order adaptive observer for sensorless induction motor control in railway traction systems under low-switching frequency. IEEE Journal of Emerging and Selected in Topic Power Electronics, 7(4), 2333-2345, 2019. https://doi.org/10.1109/JESTPE.2019.2898875

[2] M. Celebi and M. Toren, Heuristic algorithm-based estimation of rotor resistance of an induction machine by slot parameters with experimental verification. Turkish Journal of Electrical Engineering \& Computer Sciences, 25, 3553-3568 2017. https://doi.org/ 10.3906 /elk-1511-232

[3] A. Pal, S. Das, and A. K. Chattopadhyay, An improved rotor flux space vector based MRAS for field-oriented 
control of induction motor drives. IEEE Transaction on Power Electronics, 33(6), 5131-5141, 2018. https://doi.org/10.1109/TPEL.2017.2657648.

[4] E. Zerdali and E. C. Mengüç, Novel complex-valued stator current-based MRAS estimators with different adaptation mechanisms. IEEE Transaction on Instrumentation and Measurement, 68(10), 3793-3795, 2019. https://doi.org/10.1109/TIM.2019.2932161.

[5] J. You, W. Wu, and Y. Wang, An Adaptive Luenberger Observer for Speed-Sensorless Estimation of Induction Machines. 2018 Annual American Control Conference (ACC), pp. 307-312, Milwaukee, WI, USA, 27-29 June 2018. https://doi.org/10.23919/ACC.2018. 8431006.

[6] Y. Zhang, Z. Yin, J. Liu, and X. Tong, Design and implementation of an adaptive sliding-mode observer for sensorless vector controlled induction machine drives. Journal of Electrical Engineering and Technology, 13(3), 1304-1316, 2018. https://doi.org/ 10.5370/JEET.2018.13.3.1304.

[7] R. Demir and M. Barut, Novel hybrid estimator based on model reference adaptive system and extended Kalman filter for speed-sensorless induction motor control. Transactions of the Institute of Measurement and Control, 40(13), 3884-3898, 2018. https://doi.org/ 10.1177/0142331217734631.

[8] M. Barut, R. Demir, E. Zerdali, and R. Inan, Real-Time implementation of bi input-extended Kalman Filterbased estimator for speed-sensorless control of induction motors. IEEE Transactions on Industrial Electronics, 59(11), 4197-4206, 2012. https://doi.org/ 10.1109/TIE.2011.2178209.

[9] R. Yildiz, M. Barut, and R. Demir, Extended Kalman filter based estimations for improving speed-sensored control performance of induction motors. IET Electric Power Applications, 14(12), 2471-2479 2020. https:// doi.org/110.1049/iet-epa.2020.0319.

[10] M. Barut, S. Bogosyan, and M. Gokasan, Switching EKF technique for rotor and stator resistance estimation in speed sensorless control of IMs. Energy Conversion and Management, 48(12), 3120-3134, 2007. https://doi.org/10.1016/j.enconman.2007.04.026

[11] M. Barut, S. Bogosyan, and M. Gokasan, Experimental evaluation of braided EKF for sensorless control of induction motors, IEEE Transactions on Industrial Electronics, 55(2), 620-632, 2008. https://doi.org/ 10.1109/TIE.2007.911956.

[12] X. Zhang, Sensorless induction motor drive using indirect vector controller and sliding-mode observer for electric vehicles. IEEE Transactions on Vehicular Technology, 62(7), 3010-3018, 2013. https://doi.org/ 10.1109/TVT.2013.2251921.

[13] B. Wang, C. Luo, Y. Yu, G. Wang, and D. Xu, Antidisturbance speed control for induction machine drives using high-order fast terminal sliding-mode load torque observer. IEEE Transaction on Power Electronics, 33(9), 7927-7937, 2018. https://doi.org/ 10.1109/TPEL.2017.2765522.

[14] E. Zerdali, R. Demir, and M. Barut, Speed-Sensorless FCS-PTC Based Induction Motor Drive Capable of Disturbance Rejection. 2020 2nd Global Power, Energy and Communication Conference (GPECOM), pp. 170175, Izmir, Turkey, 20-23 October 2020 https://doi.org /10.1109/GPECOM49333.2020.9247917

[15] E. Zerdali and R. Demir, Speed-sensorless predictive torque controlled induction motor drive with feedforward control of load torque for electric vehicle applications. Turkish Journal of Electrical Engineering \& Computer Sciences, 29, 223-240 2021, https://doi .org/10.3906/elk-2005-75.

[16] R. Demir and E. Zerdali, Improved Speed-Sensorless Field-Oriented Controlled Induction Motor Drive With Feed-Forward Control of Load Torque. 2. International Turkic World Congress on Science and Engineering, (TURK-COSE 2020) pp. 525-532 Nur-Sultan, Kazakhstan, 14-15 November 2020.

[17] R. Inan, R. Demir ve M. Barut, Asenkron motorun karma kestirici tabanlı hız-algılayıcılı doğrudan vektör kontrolü. Ömer Halisdemir Üniversitesi Mühendislik Bilimleri Dergisi, 7(2), 612-623 2018. https://doi.org/ 10.28948/ngumuh.443233.

[18] J. Holtz, Sensorless control of induction motorsperformance and limitations. Proceedings of the 2000 IEEE International Symposium on Industrial Electronics. (ISIE 2000), pp. PL12-PL20, Cholula, Puebla, Mexico, 4-8 December 2000. https://doi .org/10.1109/ISIE.2000.930474. 